\title{
Study on the driving cycle construction for city hybrid bus
}

\author{
Jiankun Peng ${ }^{1, a}$, Deng Pan ${ }^{1, b}$ and Hongwen $\mathrm{He}^{1, \mathrm{c} *}$ \\ ${ }^{1}$ National Engineering Laboratory for Electric Vehicles, Beijing Institute of Technology, Beijing \\ 100081, China \\ apengjiankun870719@163.com, bhdpandeng@163.com, chwhebit@bit.edu.cn
}

Keywords: driving cycle, kinematic sequences, principal component, cluster analysis

\begin{abstract}
The driving date of hybrid bus which can be represent the city driving cycle is fully collected, the original data is divided into several kinematic sequences according to the velocity information, and the city hybrid bus driving cycle is built by using principal component analysis and cluster analysis. The results show that, the correlation coefficient of constructed driving cycle and original dada reaches $97 \%$, the actual operation situation of hybrid bus can be fully reflected, this study has good practical value.
\end{abstract}

\section{Introduction}

The emission and fuel economy of city hybrid bus are greatly impacted by city driving cycle [1], the control strategy development of hybrid vehicle is often based on driving cycle as the design basis [2]. The prevailing driving cycles are developed for traditional fuel vehicle, such as the European NEDC cycle, the America New York Bus cycle, the Japan 10-15 cycle and China typical urban cycle [3], but these cycles can not adequately represent the operation characteristics of the hybrid vehicle. Therefore, the development of hybrid city vehicle driving cycle is necessary.

In this paper. The development process and data processing method of driving cycle is described in detail, and the hybrid bus driving cycle of Zhengzhou city can be combined by applying statistical theory of probability distributions, the design basis for developing and researching hybrid city bus which is in line with the characteristics of Zhengzhou city bus can be provided.

\section{The original traffic data acquisition}

The original vehicle traffic data can reflect urban road traffic condition and vehicle driving characteristics, which is the data source for driving cycle construction. Therefore, the traffic data as the basis for driving cycle, the original traffic data collected more accurate and detailed, then through the analysis of these original data can get the vehicle driving characteristics which are more real and objective, the resulting driving cycle are more representative and pertinence. The two hybrid bus routes of Zhengzhou city bus are chosen for the study objective of traffic acquisition experiment, the experiment last two weeks and collected more than 600 thousands traffic data which is provided the data assurance for the late study.

Table 1 Bus route profile of data acquisition

\begin{tabular}{lll}
\hline Route & 1 & 2 \\
\hline One-way distance & $15 \mathrm{Km}$ & $21 \mathrm{Km}$ \\
\hline Stops & 27 & 36 \\
\hline Single run time & $2.5 \sim 3 \mathrm{~h}$ & $3.5 \sim 4 \mathrm{~h}$ \\
\hline \multirow{2}{*}{ Acquisition time } & $\begin{array}{l}7: 00 \sim 9: 00 ; \\
16: 00 \sim 18: 00\end{array}$ & $10: 00 \sim 12: 00 ;$ \\
\hline
\end{tabular}

The required measured parameters of experiment include: vehicle velocity, vehicle instantaneous acceleration, road slope. The experiment equipment is OXTS Inertial+. The experiment acquisition system is shown in Fig. 1. 


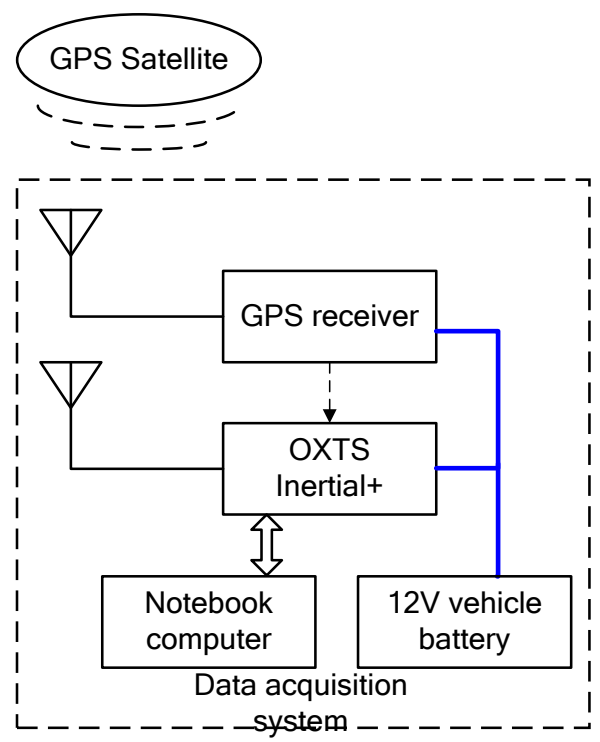

Figure 1 GPS and inertial navigation combined system

OXTS Inertial + measurement accuracy of the parameters are listed in Tab. 2:

Table 2 Measurement accuracy of the parameters

\begin{tabular}{llllll}
\hline \multirow{2}{*}{$\begin{array}{l}\text { Postion } \\
\text { accuracy }\end{array}$} & \multirow{2}{*}{ Velocity accuracy } & \multicolumn{4}{l}{ Acceleration accuracy } \\
\cline { 2 - 6 } & & Deviation & Linearity & Scale factor & Range \\
\hline $2 \mathrm{~cm} 1 \sigma$ & $0.05 \mathrm{~km} / \mathrm{hRMS}$ & $10 \mathrm{~mm} / \mathrm{s}^{2} 1 \sigma$ & $0.01 \%$ & $0.1 \% 1 \sigma$ & $100 \mathrm{~m} / \mathrm{s}^{2}$ \\
\hline
\end{tabular}

This paper adopts OXTS Inertial+ system to measure vehicle velocity and acceleration. Compare with the traditional method of reading ABS velocity signals, this method can avoid tire pressure and slip on the impact of velocity measurement, improve the measurement accuracy. Therefore, the data sampling frequency is properly increased to retain more details in the velocity sequence. $5 \mathrm{~Hz}$ sampling frequency was selected in the experiment.

\section{Driving cycle construction}

The so-called kinematic sequence refers from an idling state to the next idling state, a complete kinematic sequence typically contains idling, acceleration, deceleration and constant speed these four running states.

The steps of kinematic sequences which based on the analysis method of multi-characteristic parameters are as follows: Firstly all kinematic sequences are extracted from the original data and its characteristic parameters are calculated, secondly the characteristic parameters of kinematic sequences are dimension reduced by using principal component analysis, lastly the kinematic sequences which have similar characteristic parameters are clustered into a category through combining with the cluster analysis, and the final driving cycle can be consisted of the several kinematic sequences from the same category with a random selection [4,5].

\subsection{Analysis method of kinematic sequences}

\subsubsection{The kinematic sequences dimidiation and its characteristic parameters selection}

According to the definition of kinematic sequences, 2055 sequences are got from original data. Due to the research objective is hybrid bus, the velocity threshold value of idle sequence is taken $0.7 \mathrm{~km} / \mathrm{h}$, and the threshold value of acceleration is taken $0.15 \mathrm{~m} / \mathrm{s} 2$.

13 parameters are selected for principal component analysis and cluster analysis of kinematics sequences. The parameters are shown in the Tab. 3.

\subsubsection{Characteristic parameters matrix of kinematic sequences and its standardization}

After the statistical computation for characteristic parameters of each kinematic sequence, fill in the table as follows, and constitute the $2055 \times 13$ characteristic parameters matrix of kinematic sequences. 
Table 3 The characteristic parameters of kinematic sequences

\begin{tabular}{cll}
\hline Parameters & \multicolumn{1}{c}{ Meaning } & Unit \\
\hline $\mathrm{N}$ & The total number of sampling points & \\
$\mathrm{N}_{\mathrm{a}}$ & The sampling points of acceleration sequences & \\
$\mathrm{N}_{\mathrm{d}}$ & The sampling points of deceleration sequences & $\mathrm{Km} / \mathrm{h}$ \\
$\mathrm{N}_{\mathrm{c}}$ & The sampling points of uniform sequences & $\mathrm{Km} / \mathrm{h}$ \\
$\mathrm{N}_{\mathrm{i}}$ & The sampling points of stop sequences & $\mathrm{Km} / \mathrm{h}$ \\
$\mathrm{V}_{\max }$ & The maximum velocity & $\mathrm{m} / \mathrm{s}^{2}$ \\
$\mathrm{~V}_{\mathrm{sd}}$ & The average velocity & $\mathrm{m} / \mathrm{s}^{2}$ \\
$\alpha_{\max }$ & The standard deviation of velocity & $\mathrm{m} / \mathrm{s}^{2}$ \\
$\alpha_{\min }$ & The maximum acceleration & $\mathrm{m} / \mathrm{s}^{2}$ \\
$\alpha_{\mathrm{sd}}$ & The minimum acceleration & $\mathrm{m} / \mathrm{s}^{2}$ \\
$\alpha_{\mathrm{a}}$ & The standard deviation of acceleration & The average acceleration of acceleration sequences \\
$\alpha_{\mathrm{d}}$ & The average acceleration of deceleration sequences &
\end{tabular}

Table 4 Characteristic parameter matrix

\begin{tabular}{c|c|c|c|c|c|}
\hline No. & $\mathrm{N}$ & $\mathrm{N}_{\mathrm{a}}$ & $\cdots$ & $\alpha_{\mathrm{a}}$ & $\alpha_{\mathrm{d}}$ \\
\hline 1 & 185 & 53 & $\cdots$ & 0.503 & -0.885 \\
\hline 2 & 242 & 60 & $\cdots$ & 0.797 & -0.555 \\
\hline 3 & 268 & 146 & $\cdots$ & 0.524 & -0.907 \\
\hline 4 & 29 & 2 & $\cdots$ & 0.745 & -0.189 \\
\hline$\cdots$ & $\cdots$ & $\cdots$ & $\cdots$ & $\cdots$ & $\cdots$ \\
\hline 2054 & 571 & 273 & $\cdots$ & 0.530 & -0.759 \\
\hline 2055 & 158 & 69 & $\cdots$ & 0.707 & -0.711 \\
\hline
\end{tabular}

The characteristic parameters have the difference of dimension due to the various units, it will result in the dispersion degree of the parameters is high, and the impact on the total variance of an indicator which has a greater variance is larger than any other indicators. Therefore, the characteristic parameters matrix of kinematic sequences should be standardized, namely each column mean is 0 , and each column variance is 1 .

If set the $\mathrm{i}$-th row, $\mathrm{j}$-th column element of the kinematic sequences parameters matrix is $\mathrm{x}_{\mathrm{ij}}$, the element of the corresponding position after normalization is $\mathrm{y}_{\mathrm{ij}}$, the normalization process can be expressed as:

$$
\mathrm{y}_{\mathrm{ij}}=\frac{\mathrm{x}_{\mathrm{ij}}-\overline{x_{j}}}{S_{j}}
$$

where, $\overline{x_{j}}=\frac{1}{\mathrm{n}} \sum_{\mathrm{i}=1}^{\mathrm{n}} x_{\mathrm{ij}}, \quad \mathrm{S}_{\mathrm{j}}=\sqrt{\frac{1}{n-1} \sum_{i=1}^{n}\left(x_{i j}-\overline{x_{j}}\right)^{2}}$

Standardized results are shown in Tab. 5.

Table 5 Normalization for characteristic parameter matrix

\begin{tabular}{c|c|c|c|c|c|}
\hline No. & $\mathrm{N}$ & $\mathrm{N}_{\mathrm{a}}$ & $\cdots$ & $\alpha_{\mathrm{a}}$ & $\alpha_{\mathrm{d}}$ \\
\hline 1 & -0.326 & -0.314 & $\cdots$ & 0.073 & -1.014 \\
\hline 2 & -0.058 & -0.229 & $\cdots$ & 1.158 & -0.051 \\
\hline 3 & 0.064 & 0.809 & $\cdots$ & 0.150 & -1.076 \\
\hline 4 & -1.060 & -0.930 & $\cdots$ & 0.966 & 1.017 \\
\hline$\cdots$ & $\cdots$ & $\cdots$ & $\cdots$ & $\cdots$ & $\cdots$ \\
\hline 2054 & 1.490 & 2.343 & $\cdots$ & 0.173 & -0.645 \\
\hline 2055 & -0.453 & -0.121 & $\cdots$ & 0.825 & -0.505 \\
\hline
\end{tabular}

\subsubsection{Principal component analysis}

Principal component analysis can transform the original many corresponding indexes into the few independent comprehensive indexes. Principal component analysis can meet in the premise of as much as possible to maintain the original data information, the original variable linear combination 
transform to a new variable, and new variables independent to each other. Principal component analysis plays a major role of dimensionality reduction and simplification of data structure.

Several principal components can be obtained based on principal component analysis, a new sampling score matrix of principal component which can represent more than $90 \%$ of the original sampling information are compressed from the original overall sampling matrix. The new matrix will be used for cluster analysis to classify the overall sampling of kinematic sequences.

Set $\Sigma$ is the covariance matrix of $X=\left(X_{1}, X_{2}, \cdots, X_{p}\right)^{T}$, the characteristic parameter of $\Sigma$ and corresponding orthogonal unit are $\lambda_{1} \geq \lambda_{2} \geq \cdots \geq \lambda_{p} \geq 0$ and $e_{1}, e_{2}, \cdots, e_{p}$, thus the $i$-th principal component of $X$ is:

$Y_{\mathrm{i}}=e_{\mathrm{i}}^{T} X=e_{i 1} X_{1}+e_{i 2} X_{2}+\cdots e_{i p} X_{p},(i=1,2, \cdots, p)(2)$

According to the above equation, the 13 components contribution rates of kinematic sequences characteristic parameter matrix after standardization are obtained.

Seen from the Tab. 6, the cumulative contribution rate of first four main components has reached $94.33 \%$, the first four principal components can be used to compress the original overall sample matrix to a new sample which maintains more than $90 \%$ of the original sample information according to Equation 2, then principal component score matrix is obtained. This matrix will be used for cluster analysis.

Table 6 Contribution of principal component

\begin{tabular}{llll}
\hline No. & Variance & Contribution & $\begin{array}{l}\text { Cumulative } \\
\text { contribution }\end{array}$ \\
\hline 1 & 8.6229 & $66.33 \%$ & $66.33 \%$ \\
2 & 1.8227 & $14.02 \%$ & $80.35 \%$ \\
3 & 1.1413 & $8.78 \%$ & $89.13 \%$ \\
4 & 0.6753 & $5.19 \%$ & $94.33 \%$ \\
5 & 0.2721 & $2.09 \%$ & $96.42 \%$ \\
6 & 0.2049 & $1.58 \%$ & $97.99 \%$ \\
7 & 0.0866 & $0.67 \%$ & $98.66 \%$ \\
8 & 0.0604 & $0.46 \%$ & $99.13 \%$ \\
9 & 0.0559 & $0.43 \%$ & $99.56 \%$ \\
10 & 0.0318 & $0.24 \%$ & $99.80 \%$ \\
11 & 0.0189 & $0.15 \%$ & $99.95 \%$ \\
12 & 0.0069 & $0.05 \%$ & $100.00 \%$ \\
13 & $-1.804 \mathrm{e}-16$ & $0.00 \%$ & $100.00 \%$ \\
\hline
\end{tabular}

\subsubsection{The cluster analysis of kinematic sequences}

Cluster analysis the method that research individual classification according to the thing itself characteristic. The individual in the same class have greater similarity and difference in the different classes is the principal of cluster analysis. Cluster analysis includes a wide range contents, it can have a variety of methods to do data classification. Dynamic cluster analysis is adopted in this paper.

Dynamic cluster analysis also known as average value cluster method. This method can automatically determine the center location of $\mathrm{k}$ group. Calculating the distance of each record to the center location, then adding these records to a group according to the principal of nearest distance. Recalculating the center location of the new group, and repeating the above steps until it reached a certain standard. This method is fast and suitable for large amounts of data classification.

About selecting accumulation point, this paper carried out according to the principle of minimum and maximum. The $\mathrm{n}$ samples were divided into $\mathrm{k}$ categories, firstly select two farthest distance samples among all samples as the two initial accumulation points, so

$$
d\left(x_{11}, x_{12}\right)=d_{11,12}=\max \left\{d_{i j}\right\}
$$

Then, select the third accumulation point $x 13$, so as to satisfy the equation 4 :

$$
\min \left\{d\left(x_{13}, x_{1 n}\right), n=1,2\right\}=\max \left\{\min \left[d\left(x_{j}, x_{1 n}\right), n=1,2\right]\right\}
$$


And so on, according to the same principal to select until find $\mathrm{k}$ initial accumulation pints $L^{(1)}=\left\{x_{11}, x_{12}, \cdots, x_{1 k}\right\}$. Calculate the distance among the selected point and other points, and all the sampling points with the initial accumulation point as the center are divided into $\mathrm{k}$ disjoint sets based on nearest principal, denoted as $G^{(1)}=\left\{G_{1}^{(1)}, G_{2}^{(1)}, \cdots, G_{k}^{(1)}\right\}$. Then, on the basis of $G^{(1)}$, calculating a new cluster point $L^{(2)}=\left\{x_{21}, x_{22}, \cdots, x_{2 k}\right\}$ :

$$
x_{2 i}=\frac{1}{n_{i}} \sum_{x_{i} \in G_{i}^{1}} x_{l}, i=1,2, \cdots, k
$$

where $x i$ is all sampling points in the current cluster set.

Thus the second round of clustering points can be calculated, a new accumulation point can be regarded as the gravity or the midpoint of its cluster set. Then from L(2), re-devising the sampling points into k disjoint sets, denoted $G^{(2)}=\left\{G_{1}^{(2)}, G_{2}^{(2)}, \cdots, G_{k}^{(2)}\right\}$. Such repeated calculation continues until $L^{(m)}=L^{(m+1)}$.

\subsubsection{Driving cycle combination}

The classification of cluster sets is set to 3, the kinematic sequences duration and the time proportion of these three cluster sets are shown in Fig. 2.

Figure 2 Duration of total kinematic sequences

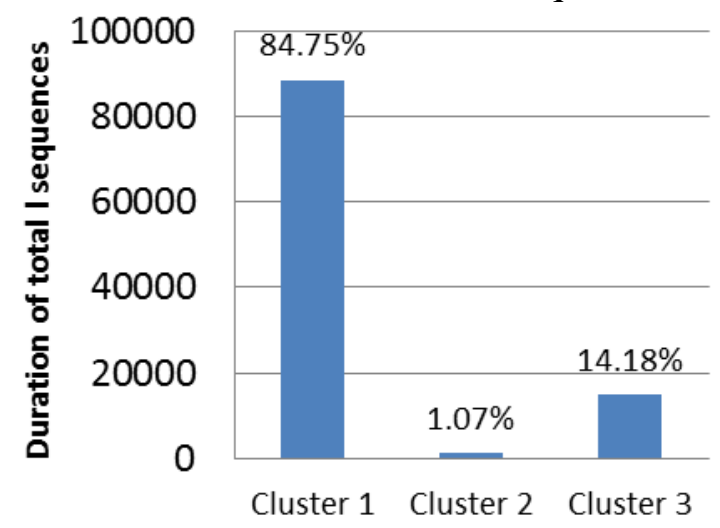

The duration of designed driving cycle is defined to 1200s, the duration of the 3 cluster classes are calculated according to the time proportion, then the kinematic sequences are randomly selected from each cluster sets until the corresponding cluster duration meet the requirements. The ultimate fusion driving cycle are shown in Fig. 3.

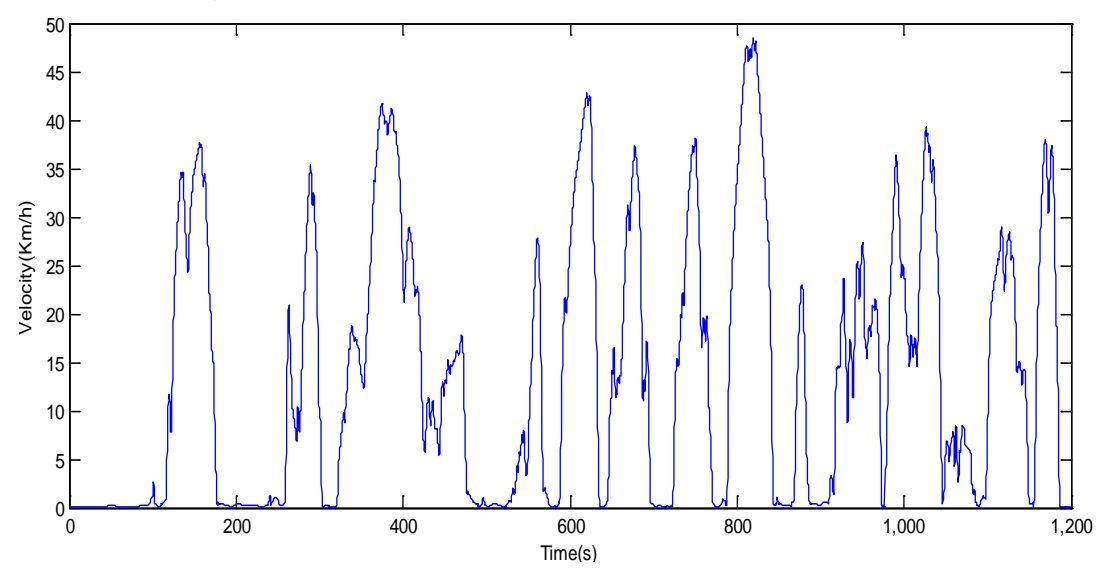

Figure 3 The ultimate fusion driving cycle

To verify the effectiveness of the constructed driving cycle, the characteristic parameters of the driving cycle are compared with the original data, the comparison results are shown in Tab. 7. 
Table 7 Comparison of statistical value

\begin{tabular}{lcc}
\hline \multicolumn{1}{c}{ Parameters } & Original data & Driving cycle \\
\hline Proportion of stop time & $24.09 \%$ & $32.51 \%$ \\
Proportion of uniform time & $19.91 \%$ & $16.80 \%$ \\
Proportion of acceleration time & $30.31 \%$ & $27.30 \%$ \\
Proportion of deceleration time & $25.69 \%$ & $23.39 \%$ \\
Standard deviation of velocity & 13.67 & 13.742 \\
Maximum velocity & 53.762 & 48.489 \\
average velocity & 14.8962 & 13.688 \\
Minimum acceleration & -5.399 & -2.777 \\
Maximum acceleration & 4.507 & 2.459 \\
Standard deviation of acceleration & 0.5944 & 0.542 \\
\hline
\end{tabular}

The correlation coefficient is an intensity metric that describes direct linear relationship between two variables. The calculation expression is expressed as:

$$
\rho=\frac{\operatorname{Cov}(X, Y)}{\sqrt{\operatorname{Var}(X) \operatorname{Var}(Y)}}
$$

where $\operatorname{Cov}(X, Y)$ is the covariance of variable $\mathrm{X}$ and $\mathrm{Y}, \operatorname{Var}(X), \operatorname{Var}(Y)$ are variance of variable $\mathrm{X}$ and $\mathrm{Y}$, respectively.

According to Equation 6, the calculated correlation coefficient is 0.97, the constructed road driving cycling with the original experimental data are highly correlated, it indicated that the build cycle is objective and effective.

\section{Conclusions}

(1) In this paper, several kinematic sequences are obtained by dividing Zhengzhou city hybrid bus driving data, and the driving cycle of hybrid bus for Zhengzhou city are constructed by using principal component and cluster analysis.

(2) The constructed driving cycle compared with the original velocity acquisition data, the proportion of characteristic parameters are the basically same, the correlation coefficient reaches $97 \%$, indicating that the construction method for driving cycle in this paper is correct, the constructed driving cycle of hybrid bus for Zhengzhou City, is true and reliable.

\section{Acknowledgments}

This work was supported by the National High Technology Research and Development Program of China (2013BAG05B00) in part, the Program for New Century Excellent Talents in University (NCET-11-0785) and Beijing Institute of Technology Post Graduate Students Innovation Foundation in part. The author would also like to thank the reviewers for their corrections and helpful suggestions.

\section{References}

[1] Fontaras G, Pistikopoulos P, Samaras Z. Experimental evaluation of hybrid vehicle fuel economy and pollutant emissions over real-world simulation driving cycles [J]. Atmospheric environment, 2008, 42(18): 4023-4035.

[2] Amjad S, Neelakrishnan S, Rudramoorthy R. Review of design considerations and technological challenges for successful development and deployment of plug-in hybrid electric vehicles [J]. Renewable and Sustainable Energy Reviews, 2010, 14(3): 1104-1110.

[3] Silva C, Ross M, Farias T. Evaluation of energy consumption, emissions and cost of plug-in hybrid vehicles [J]. Energy Conversion and Management, 2009, 50(7): 1635-1643. 
[4] Shi Qin, Zheng Yubo, Jiang Ping. A Research on Driving Cycle of City Roads Based on Microtrips [J]. Automotive Engineering, 2011, 33(3): 256-261. (in Chinese)

[5] Hung W T, Tong H Y, Lee C P, et al. Development of a practical driving cycle construction methodology: A case study in Hong Kong[J]. Transportation Research Part D: Transport and Environment, 2007, 12(2): 115-128. 